\title{
Aus anderen Fachzeitschriften
}

Online publiziert: 16. Februar 2016

(C) Springer-Verlag Berlin Heidelberg 2016

Inderbritzin, J., Rombini, S., Kellerhals, M. (2015): Untersuchungen von Präferenzen und deren Einflussfaktoren für ausgewählte Apfelsorten in der Schweiz

Mitteilungen Klosterneuburg 65, 107-120

Ausgewertet wurden 2 Konsumententests mit bekannten und neueren Apfelsorten:

'Gala, Schniga', 'Braeburn, Rosabel', 'Mairac', 'Ladina', 'Natyra', 'Jazz', 'Opal' (UEB32642), 'Galiwa' (CH101), 'Lucy’ (UEB-I-181/3), 'Greenstar', 'Renoir'. Geschmackliche und optische Akzeptanz der Sorten wurde mit den Faktoren Alter, Geschlecht, Konsum- und Einkaufsverhalten, Geschmacksvorlieben und einer mitunter zu beobachtende Ablehnung von Neuem (Neophobie) in Verbindung gebracht. Erstaunlich dabei war, dass trotzdem die neuen Sorten 'Natyra', 'Opal', 'Jazz' und 'Mairac' besser abschnitten als 'Gala' und 'Braeburn', was Tendenzen zur Neophobie widerspricht, obwohl ermittelt wurde, dass je älter die Probanden waren, die Lust, Neues auszuprobieren, abnahm. Man führte das auf sinkendes Geruchs- und Geschmacksvermögen älterer Menschen zurück, was zu einer gewissen Gleichgültigkeit bei Apfelkauf führen könnte. Für das Gesamtergebnis hatte das aber kaum Einfluss. Bestimmte Geschmacksvorlieben beeinflussten das Ergebnis signifikant, bei der Gruppe mit Vorliebe für säuerliche Äpfel schnitt 'Jazz' besser ab, bei der süß bevorzugenden Gruppe lag 'Galiwa' vorn. Ebenso bestanden Unterschiede zwischen Männern und Frauen vor allem in der Beurteilung des Aussehens. Die sehr aufwendige Auswertung führte zu der Schlussfolgerung, dass eine Segmentierung der Konsumenten nach süßen oder sauren Vorlieben zu einer Optimierung der Vermarktung bestimmter Sorten führen könnte.

M. Fischer, Dresden-Pillnitz
Malavolte, C. (2015): OBC guidelines for intergrated production of grapes: lessons to learn and experiences to share between organic and integrated production (OBC Richtlinien für integrierte Produktion von Trauben: Erfahrungsaustausch zu Schnittstellen von organischer und integrierter Produktion)

Mitteilungen Klosterneuburg 65, 81

Ref. nach einem Vortrag auf der Konferenz über Innovative Krankheitskontrollstrategien für Europas organisch und integriert produzierenden Weinanbau, Wien, Januar 2015.

IP wird als eine unter ökologischen, sozialen und ethischen Aspekten hervorragend geeignete Methode zur Produktion qualitativ hochwertiger und sicherer Nahrungsgüter beschrieben. Sie entspricht damit den höchsten internationalen Ansprüchen für Nachhaltigkeit und Effektivität in der landwirtschaftlichen Produktion zur Erzeugung von Nahrungsmitteln im Premiumsegment. IP und Organische Produktion sollten nicht als Gegensätze gesehen werden, sondern es sollte nach Möglichkeiten gesucht werden, die für jede Seite nützlichen Aspekte des anderen zu integrieren und für die Produktion zu nutzen. Besonders sollte dies für die Verbesserung der ökologischen Infrastruktur, für das Boden- und Unkrautmanagement, für Düngung und Pflanzenschutz sowie Bewässerung gelten.

$(\mathrm{OBC}=$ International Organisation for Biological and Integrated Control).

M. Fischer, Dresden-Pillnitz. 